\section{- OPEN ACCESS}

\title{
Influence of frequency and duration of strength training for effective management of neck and shoulder pain: a randomised controlled trial
}

\author{
Christoffer H Andersen, ${ }^{1}$ Lars L Andersen, ${ }^{1}$ Bibi Gram, ${ }^{2}$ Mogens Theisen Pedersen, ${ }^{3}$ \\ Ole Steen Mortensen, ${ }^{4}$ Mette Kreutzfeldt Zebis, ${ }^{2,5}$ Gisela Sjøgaard ${ }^{2}$
}

\begin{abstract}
${ }^{1}$ National Research Centre for the Working Environment, Copenhagen, Denmark Institute of Sports Science and Clinical Biomechanics, University of Southern Denmark, Odense, Denmark 3nstitute of Exercise and Sport Sciences, University of Copenhagen, Copenhagen, Denmark

${ }^{4}$ Department of Occupational and Environmental Medicine, Bispebjerg University Hospital, Copenhagen, Denmark

${ }^{5}$ Gait Analysis Laboratory, Hvidovre Hospital, Hvidovre, Denmark
\end{abstract}

\section{Correspondence to}

Christoffer H Andersen, National Research Centre for the Working Environment, Lersø Parkalle 105,

Copenhagen 2100, Denmark; cha@nrcwe.dk

Received 29 November 2011 Accepted 17 April 2012

Published Online First 29 June 2012

\begin{abstract}
Background Specific strength training can reduce neck and shoulder pain in office workers, but the optimal combination of exercise frequency and duration remains unknown. This study investigates how one weekly hour of strength training for the neck and shoulder muscles is most effectively distributed.
\end{abstract}

Methods A total of 447 office workers with and without neck and/or shoulder pain were randomly allocated at the cluster-level to one of four groups; $1 \times 60$ (1WS), $3 \times 20$ (3WS) or $9 \times 7$ (9WS) min a week of supervised high-intensity strength training for 20 weeks, or to a reference group without training (REF). Primary outcome was self-reported neck and shoulder pain (scale 0-9) and secondary outcome work disability (Disability in Arms, Shoulders and Hands (DASH)).

Results The intention-to-treat analysis showed reduced neck and right shoulder pain in the training groups after 20 weeks compared with REF. Among those with pain $\geq 3$ at baseline $(n=256)$, all three training groups achieved significant reduction in neck pain compared with REF $(p<0.01)$. From a baseline pain rating of 3.2 (SD 2.3) in the neck among neck cases, 1WS experienced a reduction of $1.14(95 \% \mathrm{Cl} 0.17$ to 2.10$)$, 3WS 1.88 (0.90 to 2.87) and 9WS 1.35 (0.24 to 2.46) which is considered clinically significant. DASH was reduced in 1WS and 3WS only.

Conclusion One hour of specific strength training effectively reduced neck and shoulder pain in office workers. Although the three contrasting training groups showed no statistical differences in neck pain reduction, only 1 WS and 3WS reduced DASH. This study suggests some flexibility regarding time-wise distribution when implementing specific strength training at the workplace.

\section{INTRODUCTION}

Physical exercise reduces the morbidity and disability from many diseases and disorders. ${ }^{1}$ Neck pain is frequent in the working-age population and increases the risk for long-term sickness absence. ${ }^{2}$ One mode of physical exercise that has shown great promise in this regard is specific strength training. ${ }^{3}$ A Finnish study showed a $\sim 70 \%$ reduction of neck pain in response to a 1-year strength-training regime, ${ }^{4}$ and women with trapezius myalgia achieved a $\sim 80 \%$ reduction in neck pain after 10 weeks of intensive muscle-specific strength training consisting of dumbbell exercises performed $3 \times 20$ min a week. ${ }^{5}$
As little as 2 min of daily strength training have also provided modest benefits in adult office workers with frequent neck/shoulder pain. ${ }^{2}$ Altogether, many studies have shown good efficacy on neck pain from strength training. ${ }^{2-7}$ Although different strength-training protocols appears to work, the optimal frequency and duration of strength training for effective management of neck pain remain unknown. Furthermore, for physical exercise to be feasible in a workplace setting, the exercise should be easy to implement in the daily routines as this has a marked effect on training adherence. ${ }^{89}$ At some companies, a few long training sessions each week may be most suitable, whereas at other companies, several short bouts of exercise may be more feasible. Physiologically, longer and more fatiguing sessions may lead to a higher tissue turnover and thus muscle hypertrophy than shorter sessions. By contrast, shorter and less fatiguing sessions may lead to higher average training intensities and thus neural adaptations resulting in increased strength gains.

The general strength-training literature may provide further insight to the effects of different training programmes for neck pain. A metaanalysis - based on 177 resistance training studies - showed that three training days per week is optimal for efficient strength gains in untrained healthy adults without musculoskeletal disorders. ${ }^{10}$ In a workplace setting, adherence to exercise programmes is challenging, ${ }^{11}$ thus balancing the optimal physiological recommendations with practical solutions is necessary. In weight loss programmes, adherence is higher in multiple short sessions of exercise as opposed to fewer and longer sessions. ${ }^{12}$ Similarly, integration of short bouts of physical activity into organisational routine may be more feasible in a workplace setting than long exhausting training sessions. ${ }^{13}$

The aim of our study is to investigate the effect of three different time-wise combinations of strength-training programmes, all with a total of $1 \mathrm{~h}$ per week on non-specific neck and shoulder pain among office workers.

\section{METHODS}

\section{Study design}

A cluster randomised controlled trial was performed in Denmark from January to June 2010. The participants were recruited from 12 geographically different units located in major cities throughout Denmark and were cluster randomised into 
five groups. The recruitment and cluster randomisation procedure is described in an open-access protocol elsewhere. ${ }^{14}$ The 1WS group trained for $1 \mathrm{~h}$ once a week, the 3WS group trained 20 min three times a week and the 9WS group trained 7 min nine times a week. The REF was not offered any physical training, but replied to the same questionnaires as the intervention groups. The fifth group $3 \mathrm{MS}$ trained with minimal supervision and is not included in the present paper but in a paper testing the relevance of training supervision for safe and effective training. In the present paper, we tested the hypothesis: 'there is no difference between the groups 1WS, 3WS, 9WS and REF for the change in neck/shoulder pain from baseline to week 20, ${ }^{14}$ Number of participants in each group: $1 \mathrm{WS}=116,3 \mathrm{WS}=126,9 \mathrm{WS}=106$ and $\mathrm{REF}=101$. All of the participants gave their written consent to participate in the study. The local ethics committee approved the study protocol (H-C-2008-103), which qualified for registration in the ClinicalTrials.gov, number NCT01027390.

\section{Participants}

The participants were office workers employed at a national public administrative authority. We invited 2114 employees to participate in the study via an internet-based questionnaire and an invitation text went out to the prospective participants by email. Out of the invited employees, 990 replied to the questionnaire. Exclusion criteria were (1) hypertension (systolic BP $>160$, diastolic BP $>100$ ) or cardiovascular diseases, (2) symptomatic herniated disc or severe disorders of the cervical spine, (3) postoperative conditions in the neck and shoulder region, (4) history of severe trauma and (5) pregnancy (6) or other serious disease. The total number of participants included in this paper was 449 employees and of these, a total of 256 participants were pain cases (a baseline pain intensity of at least three on a $0-9$ scale). The cut-off-point of three is based on a questionnaire validation study by Kaergaard et al in which they found that $67 \%$ scoring 3 and above on a $0-9$ scale had clinical signs of myofascial pain syndrome. ${ }^{15}$

Table 1 shows that the participants in the four groups were similar with regard to gender, age, height and body mass. There was only statistical difference between completers and non completers in the baseline Disability in Arms, Shoulders and Hands (DASH) parameter $(10 \pm 14$ vs $14 \pm 19$, $\mathrm{p}<0.01)$.

In total 280 participants (62\%) replied to both the baseline and follow-up questionnaires and are termed 'completers' in the following. There was no statistical difference in any of the baseline parameters between completers and non completers (table 1). The flow of the participants is shown in figure 1.

\section{Description of Intervention}

The training groups performed the same total amount of exercises and repetitions for a total of $1 \mathrm{~h}$ per week for 20 weeks during working hours. Experienced instructors supervised every other training session.

The intervention groups performed specific strength training with five different dumbbell exercises: front raise, lateral raise, reverse flies, shrugs and wrist extension. During the 20 -week intervention, training loads were progressively increased according to the principle of periodisation and progressive overload. ${ }^{16}$ The training intensity was progressively increased from 20 repetitions maximum (RM) at the beginning of the intervention period to $8 \mathrm{RM}$ during the later phase. The training programme and exercise adjustments in case of pain is described in detail by Andersen et al. ${ }^{14}$

\section{Primary outcome measures}

Neck and shoulder pain intensity

Musculoskeletal symptoms were reported according to a modified version of the Nordic questionnaire on trouble (ache,

Table 1 Baseline characteristics for the participants

\begin{tabular}{|c|c|c|c|c|c|c|c|c|c|c|}
\hline & $1 W S$ & SD & $3 W S$ & SD & 9WS & SD & REF & SD & Total & SD \\
\hline $\mathrm{N}$ & 116 & & 126 & & 106 & & 101 & & 449 & \\
\hline Males/females & $44 / 72$ & & $39 / 87$ & & $45 / 61$ & & $42 / 59$ & & $170 / 279$ & \\
\hline Age (years) & 47 & (10) & 46 & (10) & 45 & (10) & 46 & $(10)$ & 46 & $(10)$ \\
\hline Height (cm) & 174 & (9) & 173 & (10) & 175 & (9) & 175 & (9) & 174 & (9) \\
\hline Weight (kg) & 77 & (15) & 75 & (18) & 78 & (15) & 80 & $(16)$ & 77 & $(16)$ \\
\hline BMI $\left(k g / m^{2}\right)$ & 25.2 & $(4.0)$ & 24.7 & $(4.3)$ & 25.3 & (3.7) & 26.1 & $(4.5)$ & 25.3 & $(4.1)$ \\
\hline $\begin{array}{l}\text { Pain intensity in } \\
\text { the neck previ- } \\
\text { ous } 3 \text { months } \\
\text { (scale } 0-9 \text { ) }\end{array}$ & 3.32 & $(2.25)$ & 3.13 & $(2.41)$ & 3.05 & $(2.30)$ & 3.24 & $(2.26)$ & 3.18 & $(2.30)$ \\
\hline $\begin{array}{l}\text { Pain intensity in } \\
\text { the right shoul- } \\
\text { der previous } \\
3 \text { months } \\
\text { (scale } 0-9 \text { ) }\end{array}$ & 2.22 & $(2.32)$ & 2.32 & (2.38) & 1.88 & $(2.22)$ & 2.01 & $(2.39)$ & 2.09 & (2.33) \\
\hline $\begin{array}{l}\text { Pain intensity in } \\
\text { the left shoul- } \\
\text { der previous } \\
3 \text { months } \\
\text { (scale 0-9) }\end{array}$ & 1.50 & $(2.05)$ & 1.75 & $(2.28)$ & 1.75 & $(2.18)$ & 1.50 & $(1.92)$ & 1.63 & $(2.15)$ \\
\hline $\begin{array}{c}\text { DASH (scale } \\
0-100)\end{array}$ & 12 & $(16)$ & 13 & (18) & 10 & (16) & 11 & (14) & 12 & (16) \\
\hline
\end{tabular}

DASH, Disability in Arms, Shoulders and Hands; 1WS, $1 \mathrm{~h}$ training once a week; 3WS, 20 min training three times a week; 9WS, 7 min training nine times a week; REF. reference group. 


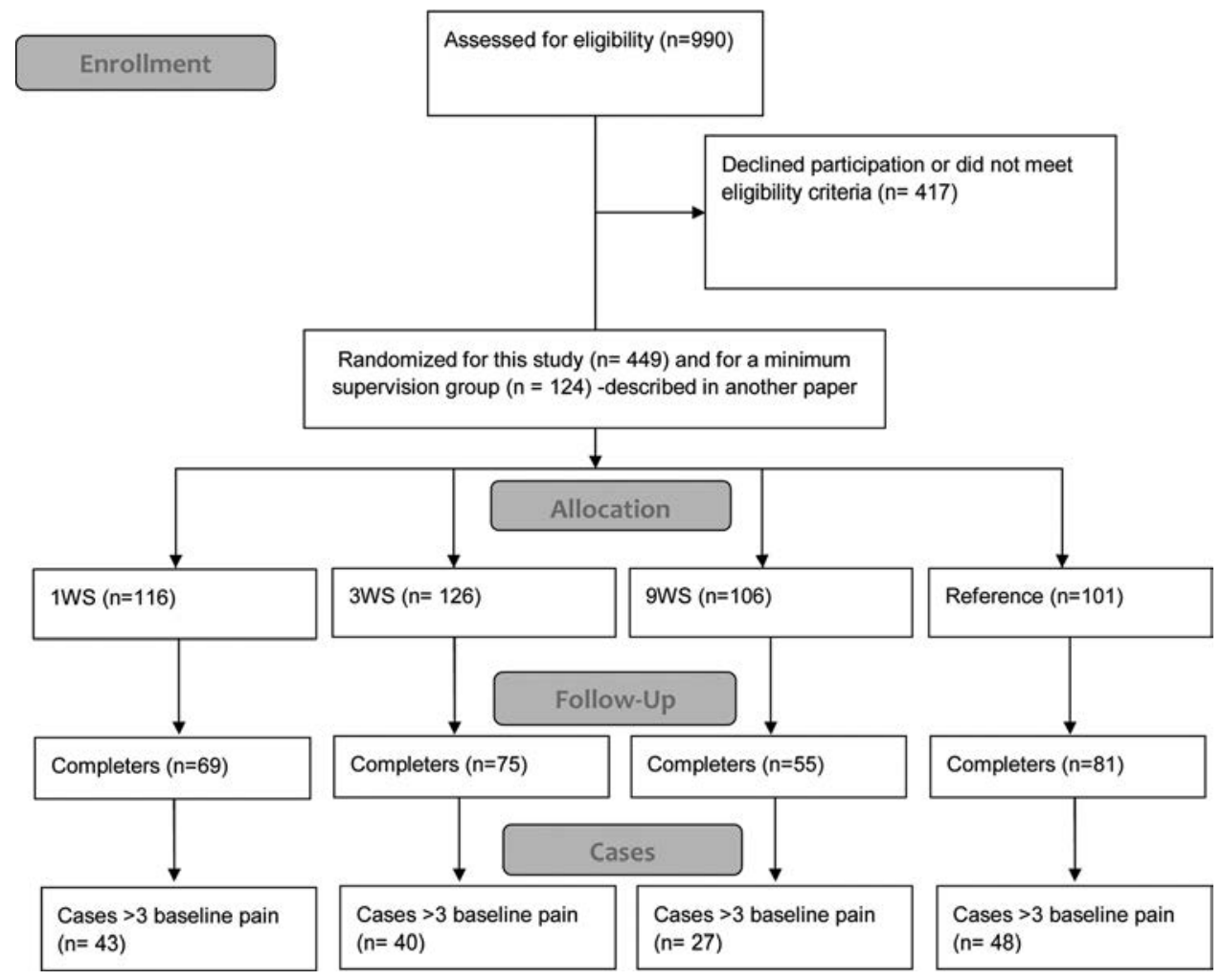

Figure 1 Flow of participants throughout the intervention.

pain or discomfort) in the neck or shoulders during the last 3 months. ${ }^{17}$ The intensity of pain in the neck and shoulder was rated subjectively on a scale ranging from 0-9 in the questionnaire, where 0 indicated 'no pain at all' and 9 indicated 'worst possible pain'.

\section{Secondary outcome measures}

\section{Disability in Arms, Shoulders and Hands}

Participants rated work disability at baseline and follow-up by the work module of the DASH questionnaire: 'in the past week did you have any difficulty:' (1) 'using your usual technique for your work?', (2) 'doing your usual work because of arm, shoulder or hand pain?', (3) 'doing your work as well as you would like?', (4) 'spending your usual amount of time doing your work?'. Participants replied on a 5-point Likert scale from 'no difficulty' to 'unable'. The DASH score was normalised on a scale of $0-100$ (by adding the four values, dividing by 4, subtracting by 1 and multiplying by 25). ${ }^{18}$

\section{Muscle strength}

In a weekly questionnaire, participants were asked to report the heaviest weight with which they were able to perform 10 repetitions of the lateral raise exercise during the last week.

\section{Adherence}

We defined adherence based on follow-up questionnaire replies on training frequency. Regular adherence was defined as participating at least $20 \mathrm{~min}$ a week during the 20 -week intervention. $^{24-619}$

\section{Statistical analyses}

Analyses were performed according to the intention-to-treat (ITT) principle that is, including all randomised participants regardless of actual participation and missing reply at follow-up. Further, we performed the analyses for completers for both the entire cohort as well as for pain cases. Imputation of missing values at follow-up were performed by adding the natural seasonal change in pain and DASH score - defined as the mean change from baseline to follow-up in REF - to the baseline value. ${ }^{20}$ In the weekly responses on 10 RM training weight in the lateral raise exercise imputation of missing values were performed by both last observation carried forward (LOCF) and first observation carried backward, and subsequently calculating the slope of the curve for each participant (ie, average weekly progression in $10 \mathrm{RM}$ training weight).

Using SPSS version 19, we performed analysis of variance to model change in pain and DASH during the last 20 weeks (follow-up value minus baseline value) in the neck and controlled for gender and baseline neck pain in the model. Correlations between baseline pain and DASH score were calculated using Spearman rank correlation. The level of significance was set to $\mathrm{p}<0.05$. Baseline results are presented as mean (SD) and changes from baseline to follow-up as means (95\% CIs) unless otherwise stated.

\section{RESULTS}

\section{Adherence}

Adherence to the training programme: $39 \%$ participated 40-60 min per week, $18 \%$ participated $20-40$ min per week. 
Thus, regular adherence - defined as participating at least 20 min a week during the 20 -week intervention - was achieved by $56 \%$ of the questionnaire respondents. In both the 3WS and the 9WS groups, regular adherence was achieved by $60 \%$, while 1 WS only achieved $49 \%$ regular adherence which was significantly lower $(p<0.05)$. Converted to total training time, the completers trained on average 789 min out of a maximally 1200 min during the 20 -week intervention. This corresponds to $66 \%$ training adherence.

Numerically, there was a higher number of dropouts in 9WS but this was not statistically different from that of the other training groups $(\mathrm{p}=0.38)$.

\section{Intention to treat analysis}

A priori hypothesis testing of main effects showed a significant group by time interaction for neck-pain intensity $(p<0.01)$, and post-hoc analyses showed a significant difference between the three training groups combined versus REF $(\mathrm{p}<0.05)$ (table 2). The three different training groups showed no statistical difference in the reduction of neck pain, and - in this analysis none of these groups were by themselves statistically different from REF.

For the right shoulder pain decreased more in the training groups combined versus REF $(p<0.05)$, while there was no effect on the left shoulder. Further, 1WS reduced right shoulder pain significantly more than REF ( $p<0.05)$.

There was a significant group by time interaction for the DASH score, with a significant difference between 1 WS and $3 \mathrm{WS}$ versus REF $(\mathrm{p}<0.005)$ and between $3 \mathrm{WS}$ and $9 \mathrm{WS}$ $(\mathrm{p}<0.05)$ (table 2).

The neck-pain cases had a mean baseline pain intensity of 4.84 (1.55) with no difference between the groups. For the 256 pain cases, (ie, a pain intensity of 3 or more at baseline) the ITT analysis showed significant group by time interaction $(p=0.05)$ (table 3). The subsequent post-hoc analysis showed greater pain reduction in the $3 \mathrm{WS}$ group compared with REF $(p<0.01)$. None of the other groups were statistically different in pain reduction.

For neck-pain cases, there was also a significant group by time interaction for DASH, with a significant difference between $3 \mathrm{WS}$ and the REF ( $p<0.01)$. 3WS also showed significant greater reduction than 9WS ( $p<0.05)$.

There was a significant correlation between baseline pain and disability for both the entire cohort (Spearmann's $\rho=0.49$, $p<0.001$ ) and the neck-pain cases (Spearmann's $\rho=0.40$, $\mathrm{p}<0.001)$.

\section{Completers}

For completers, the analysis on the entire cohort showed significant difference between the groups $(p<0.005)$. Both 1WS and 3 WS had a greater pain reduction than REF. Although $9 \mathrm{WS}$ also showed a numerically larger reduction than REF, this was only borderline significant $(p=0.07)$. There was no difference between any of the training groups.

There was a positive correlation between the baseline-tofollow-up change in pain in the neck and in the shoulders with a Spearman's correlation coefficient of 0.52 for the right shoulder and 0.35 for the left $(\mathrm{p}<0.0001)$.

There was a significant group by time interaction for DASH on the entire cohort of completers $(p<0.05)$, with a significant reduction between $1 \mathrm{WS}$ and REF $(\mathrm{p}<0.05)$ and between $3 \mathrm{WS}$ and REF ( $p<0.001)$. 3WS also showed a significant reduction compared to 9WS $(\mathrm{p}<0.05)$.

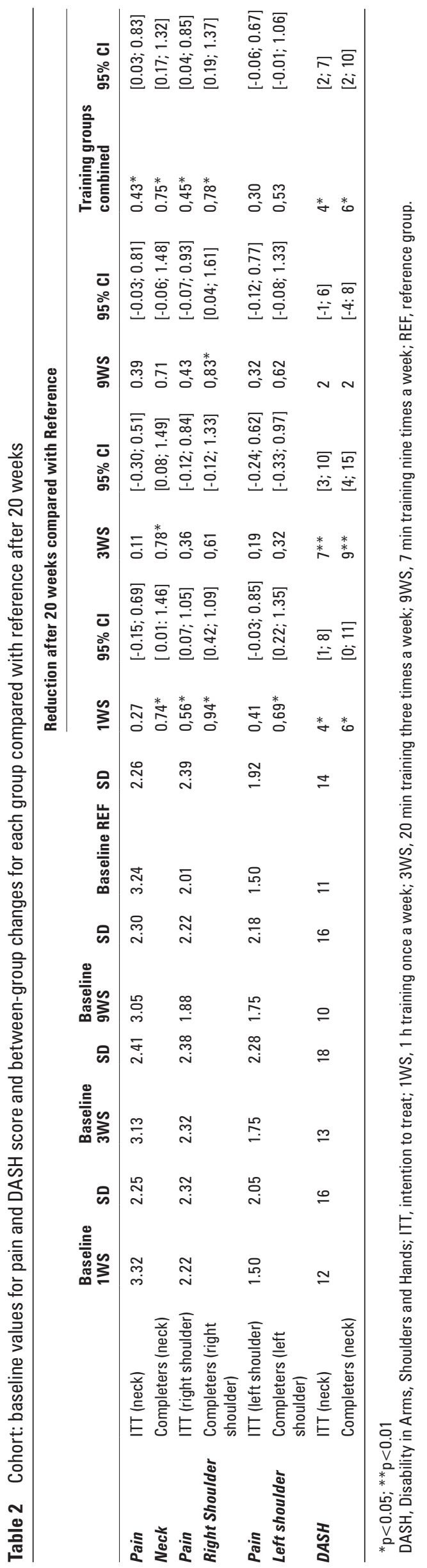




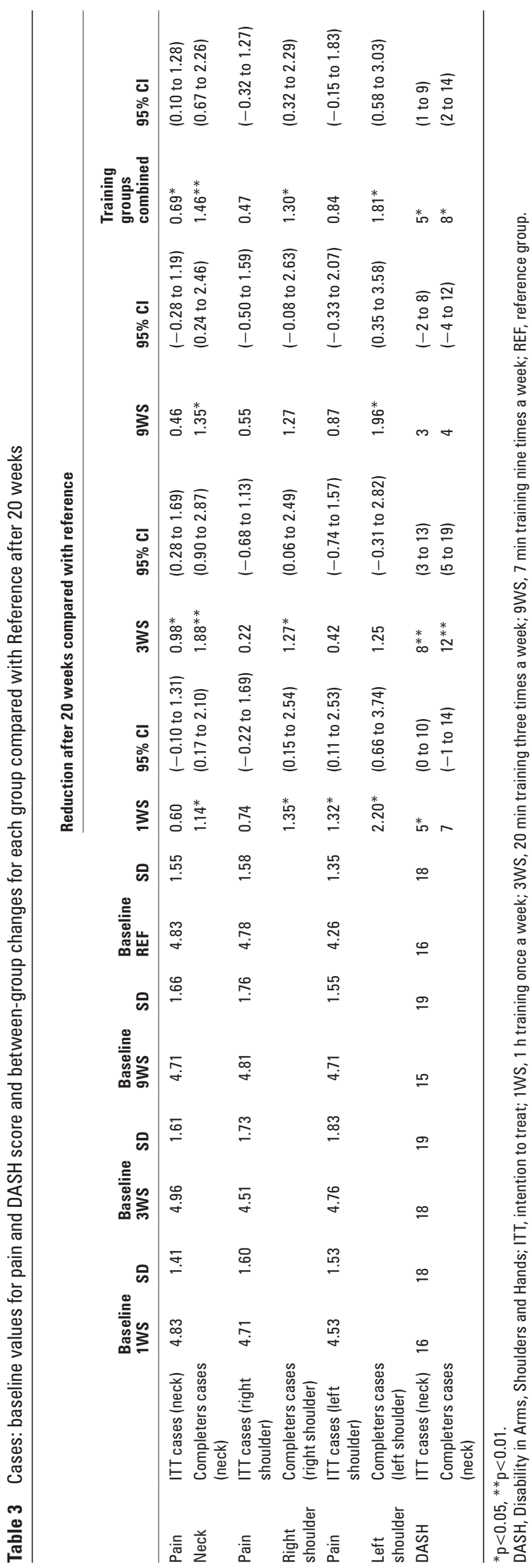

For the completers, analysis on neck-pain cases showed significant difference between the groups $(p<0.001)$. Compared with REF, a significant pain reduction was found in $3 \mathrm{WS}$ $(p<0.001), 1 \mathrm{WS}$ and $9 \mathrm{WS}(\mathrm{p}<0.05)$ (figure 2$)$. There was no statistical significant difference between training groups.

In the neck-pain cases, the percentages in each group experiencing a reduction in neck-pain intensity of more than one on a scale of $0-9$ was 36 out of 43 in 1WS ( $84 \%), 33$ out of 40 in 3 WS $(\sim 83 \%), 22$ out of 27 in 9WS ( 81\%), which was significantly higher than the 28 out of 48 in $\operatorname{REF}(\sim 58 \%)(p<0.05)$. For reductions of more than two, all training groups also fared better than REF with $31(\sim 72 \%)$ in $1 \mathrm{WS}, 30(\sim 75 \%)$ in $3 \mathrm{WS}$ and $17(\sim 63 \%)$ in 9 WS compared with $21(\sim 44 \%)$ in REF $(p<0.01)$.

For completers, there was a significant group by time interaction for DASH for the pain cases in the questionnaire responders, with a significant reduction between $3 \mathrm{WS}$ versus $\operatorname{REF}(\mathrm{p}<0.001)$.

\section{Muscle strength}

During the intervention, $10 \mathrm{RM}$ in the lateral raise exercise increased steadily with an average of $0.10 \mathrm{~kg}$ per week $(0.08$; 0.13 ) for all participants. There was no significant difference between the training groups when the LOCF procedure was performed, although there was a tendency for the increase in training weights of $1 \mathrm{WS}$ to be higher than 9WS ( $p=0.07$ ) (figure 3). Without imputation of missing values, 1WS increased their training weights on average $0.16 \mathrm{~kg}$ per week $(0.11 ; 0.21)$ which was significantly faster than $9 \mathrm{WS}$ who had an average increase of $0.07 \mathrm{~kg}$ per week $(0.03 ; 0.12)(\mathrm{p}<0.01)$. 3WS had an average of $0.12 \mathrm{~kg}$ per week $(0.09 ; 0.16)$ and tended toward increasing faster than $9 \mathrm{WS}(p=0.085)$. Baseline neck pain was not significantly correlated with the slope of the change in training weights.

\section{DISCUSSION}

The novel finding of our study is that several time-wise combinations of specific strength training can reduce neck pain in office workers. This allows certain flexibility for companies wanting to implement neck/shoulder training at the workplace.

Our study confirms that specific strength training (1) reduces the overall level of neck and rightshoulder pain among office workers and (2) has a clinical significant effect among pain cases. These findings are in accordance with previous studies. $^{2}$ 4-7 19 21-23 In our study, the mean pain reduction among the responding pain cases ranged from $47 \%$ (1WS) to $61 \%$ (3WS). Although a direct comparison between studies is difficult due to methodological differences, the marked pain reduction in our study is only surpassed with $79 \%$ reduction over 10 weeks by Andersen et al, ${ }^{5}$ who used a similar training protocol in women with trapezius myalgia, and $69 \%$ in clinical neck-pain patients over 1 year period by Ylinen et al. ${ }^{4}$

As a novel finding, our study shows that the three time-wise distributions of the strength-training programme were not significantly different in reducing pain. However, as 3 WS showed numerically higher pain reduction in both neck and shoulder pain cases as well as in the entire cohort, we cannot rule out a statistical type 2 error. Further, 1WS had lower adherence than 3WS and 9WS which indicates difficulties in fitting long sessions into the organisational routine. However, 9WS had a slower increase in training load progression and may thus be physiologically suboptimal for novice trainees.

Our analysis showed that there was positive correlation between change in neck pain and pain in either shoulder in 


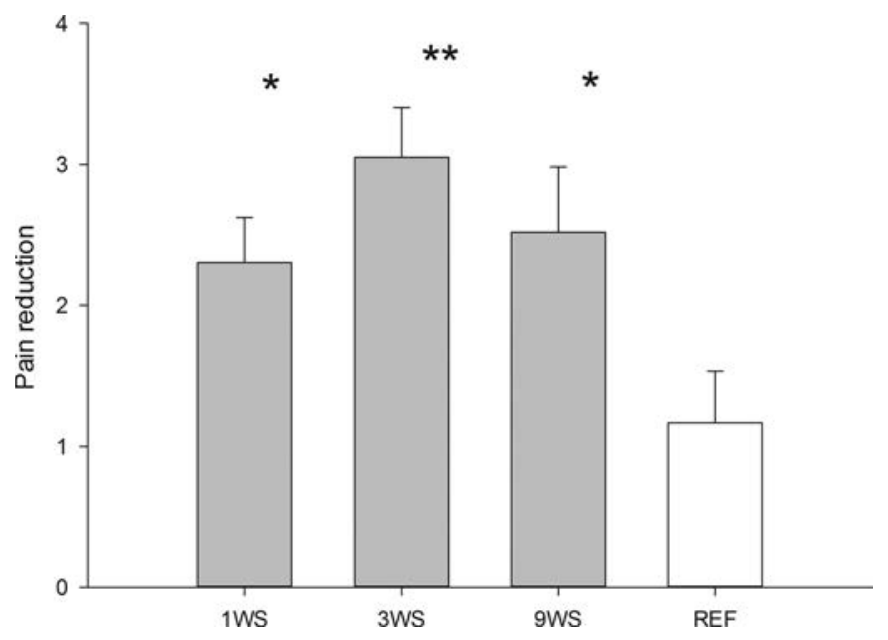

Figure 2 Change in neck pain in cases after the 20-week intervention-period. Asterisks denote difference from reference group. ${ }^{* *} p<0.01,{ }^{*} p<0.05$.

10 RM lateral raise training weight (LOCF)

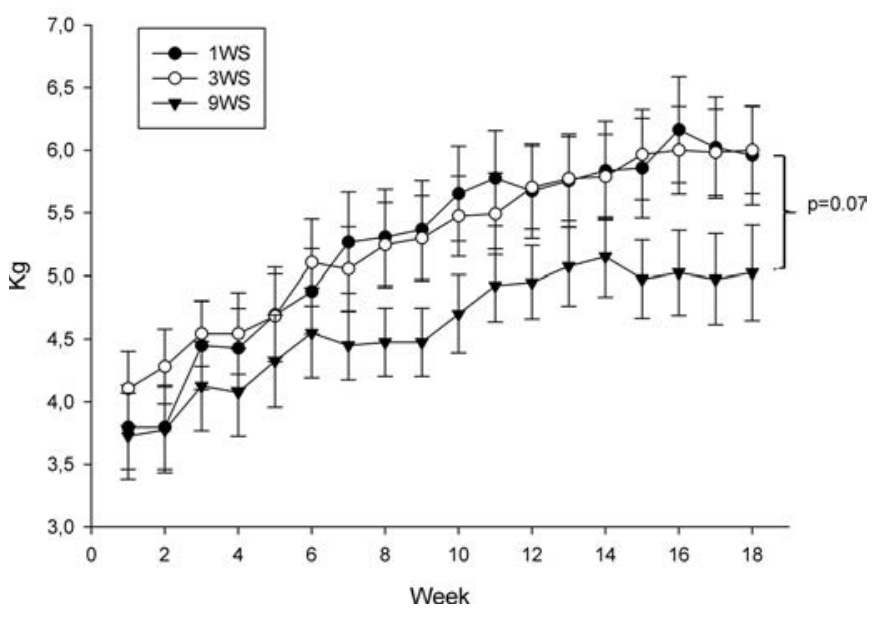

Figure $310 \mathrm{RM}$ training weights during the intervention.

accordance with previous studies. ${ }^{624}$ Chronic pain can lead to central sensitisation and referred pain in areas adjacent to the actual painful muscle, ${ }^{25}$ but the influence of these factors in the rehabilitation process - that is, pain reduction during the 20 -week training programme - is unknown. For shoulder-pain cases among completers, there were only slight differences between which interventions were most effective for the different outcomes.

In relation to DASH, $3 \mathrm{WS}$ provided the largest effect size with 9WS being generally less effective across the groups in the analyses. Thus, to improve the DASH, score training sessions may have to exceed some critical volume or level of muscle fatigue, for example, at least 20 min per training session.

To our knowledge, no previous high-quality intervention studies have compared effects of different time-wise distributions of a fixed $1 \mathrm{~h}$ per week strength-training programme with equal volume. Most training studies in relation to neck pain have used three weekly sessions. ${ }^{27}$ One recent study used five short weekly sessions, but as they used just one exercise and low training volume, comparison to previous studies is difficult. ${ }^{2}$ Regular training is important for optimal results regardless of the training programme. Rhea et al concluded in a meta-analysis on untrained healthy adults that maximum strength gains were obtained using three weekly strengthtraining sessions. This also applies to older women, that is, age seems to be of minor importance for relative strength gains. ${ }^{26}$ However, pain relief and strength gain may occur through different physiological mechanisms in different groups and optimal training frequency may therefore be different.

Even though the training frequency and duration of training sessions is roughly similar in most of the studies, training adherence varies widely. Some studies have had high training attendance: $87 \%$ (10 weeks), ${ }^{5} 85 \%$ (20 weeks), $780 \%$ (10 weeks), ${ }^{2}$ some had medium attendance: $60 \%$ (10 weeks) ${ }^{4}$ $57 \%(52 \text { weeks })^{23}$ and some had low training attendance: $39 \%$ $(12 \text { weeks })^{21}$ and $31 \%$ (12 weeks). ${ }^{23}$ In our study, the average training adherence was medium with $66 \%$ attendance over the 20 weeks. Although recruitment, screening and training procedures in this study were similar to those used by Zebis et al, ${ }^{7}$ training adherence was different between the two studies - probably due to differences in the participating companies and the circumstances under which they worked. Thus, to effectively implement training at workplaces, fitting the training sessions into the organisational routine in a flexible manner is important. Our study suggests that several combinations of a total of $1 \mathrm{~h}$ of strength training per week are effective for pain reduction. This implies a large degree of flexibility for companies and employees regarding time distribution when implementing specific strength training into a weekly schedule.

In healthy novice trainees, loads are often doubled during the initial months of training. ${ }^{27-29}$ Our study elaborates on these previous findings by showing that individuals with and without pain experience similar progression in training loads, that is, pain at baseline did not significantly halt progression in training loads. The strength-training protocol in our study was based on best-practice experience with neck-shoulder training combined with knowledge from resistance training studies in healthy individuals. ${ }^{16}$ We induced a progressive overload by periodising exercise intensity and total repetitions performed, because systematic variation of volume and intensity is effective for longterm progression in rehabilitation. ${ }^{25} 30$ This systematic variation allowed the training stimulus to remain challenging and effective throughout the 20 -week period.

\section{Strength and limitations}

As our study included both men and women from 12 geographically different units located throughout the country, the generalisability to office workers in general is high. The cluster randomisation protected against contamination between groups and provided a real-world setting where colleagues had the opportunity to do the same training programme together. However, a limitation is that not all participants replied to the follow-up questionnaire. Importantly, completers and non completers were similar in all but one of the baseline characteristics, suggesting that completers were representative of the total study population. Although statistically non significant, the 9WS group had a numerically higher dropout than the other groups, but as the dropouts have not answered the follow-up questionnaire, we can only speculate about this. One reason could be that with nine weekly sessions, some of the participants in the $9 \mathrm{WS}$ group may have experienced that having to schedule in the short sessions twice a day to be a burden. 


\section{What this study adds}

- Strength training for a total of $1 \mathrm{~h}$ per week effectively reduces neck and shoulder pain in office workers.

- Combinations of $1 \times 60,3 \times 20$ and $9 \times 7$ min per week all provide benefits in terms of pain reduction.

- Fewer and longer sessions provide larger strength gains than more frequent and shorter ones.

We imputed missing values by adding the natural seasonal change in pain and DASH score to the baseline value. ${ }^{20}$ However, all types of imputation have limitations. The current method for imputation of missing values is based on what would be expected if non completers did not train at all like the REF. This is one of the methods proposed as good practice by White et al. ${ }^{31}$ As a result, variability may be lost along with a reduction of the effect estimators of the intervention groups.

\section{CONCLUSION}

Specific strength training for the neck and shoulder muscles is an effective tool for both reduction of pain in the neck and shoulder region as well as for reduction of disability in the arms, shoulders and hands. Overall, three weekly sessions provided the most positive effects, but as long as it is manageable for the participants to adhere to, a single long session can also be effective. This implies some degree of flexibility for companies and employees regarding time-wise distribution when incorporating specific strength training in an effective manner into a weekly work schedule.

Acknowledgements Thanks to Klaus Hansen for assistance.

Contributors CHA: data collection, manuscript. LLA: study design, revision of manuscript. BG: data collection, revision of manuscript. MTP: data collection, revision of manuscript. OSM: study design, revision of manuscript. MKZ: study design, revision of manuscript. GS: study design, revision of manuscript

Funding This study was financially supported by funding from the Danish Working Environment Research Fund case number 20070014666/4. The contribution in terms of manpower allowing employees to train during work time for $1 \mathrm{~h}$ per week for 20 weeks was given by the workplaces involved.

\section{Competing interests None.}

Patient consent Obtained.

Ethics approval Local ethics committee (study protocol H-C-2008-103).

Provenance and peer review Not commissioned; externally peer reviewed.

Open Access: This is an Open Access article distributed under the terms of the Creative Commons Attribution-Non Commercial (CC BY-NC 3.0) license, which permits others to distribute, remix, adapt, build upon this work non-commercially, and license their derivative works on different terms, provided the original work is properly cited and the use is non-commercial. See: http://creativecommons.org/licenses/by-nc/3.0/

- References to this paper are available online at http://bjsm.bmjgroup.com 